\title{
Consumptive Coagulopathy and Thrombosis during severe COVID-19 infection: Potential Involvement of VWF/ADAMTS13
}

Upendra K. Katneni ${ }^{1}$, Aikaterini Alexaki ${ }^{2}$, Ryan Hunt ${ }^{2}$, Tal Schiller ${ }^{3}$, Michael DiCuccio ${ }^{4}$, Paul W. Buehler ${ }^{1}$, Juan C. Ibla ${ }^{5}$ and Chava Kimchi-Sarfaty ${ }^{2 *}$

\author{
Affiliations: \\ USA. \\ * Corresponding author: \\ Chava Kimchi-Sarfaty, Ph.D. \\ OTAT Deputy Associate Director for Research \\ Division of Plasma Protein Therapeutics \\ FDA | CBER | OTAT \\ Office: 240-402-8203 \\ E-mail: Chava.kimchi-sarfaty@fda.hhs.gov
}

${ }^{1}$ The Center for Blood Oxygen Transport and Hemostasis, Department of Pediatrics, University of Maryland School of Medicine, Baltimore Maryland, USA

${ }^{2}$ Hemostasis Branch, Division of Plasma Protein Therapeutics, Office of Tissues and Advanced Therapies, Center for Biologics Evaluation \& Research, US FDA, Silver Spring, MD, USA.

${ }^{3}$ Diabetes, Endocrinology and Metabolic Disease Unit, Kaplan Medical Center, Rehovot, Israel.

${ }^{4}$ National Center of Biotechnology Information, National Institutes of Health, Bethesda, MD,

${ }^{5}$ Department of Anesthesiology, Perioperative and Pain Medicine, Division of Cardiac Anesthesia, Boston Children's Hospital and Harvard Medical School, Boston, MA, USA. 


\begin{abstract}
Coronavirus disease of 2019 (COVID-19) is the clinical manifestation of the respiratory infection caused by severe acute respiratory syndrome coronavirus 2 (SARS-CoV-2). Following its origin as a regional outbreak in Wuhan, China, COVID-19 rapidly spread globally and eventually was designated as pandemic by the World Health Organization. Multiple studies describing the clinical characteristics of COVID-19 patients highlighted the prevalence of abnormal coagulopathy and higher incidence of thrombosis. These studies identified co-morbid inflammatory disorders as risk factors for hospitalization in SARS-CoV-2 infections. While early evidence suggested inflammatory conditions as the leading cause of abnormal coagulopathy in COVID-19 patients, the mechanisms behind progression of inflammation mediated hemostasis dysregulation to thrombotic outcomes in susceptible individuals are not well understood. In addition, a sensitive and temporal assessments of coagulation and fibrinolysis is still lacking. Von Willebrand Factor (VWF) and ADAMTS13 interactions play an important role in the maintenance of hemostasis and prevention of unwanted thrombosis. In inflammatory conditions, VWF-ADAMTS13 imbalance characterized by elevated VWF levels and inhibited and/or reduced activity of ADAMTS13 is reported. Also, an imbalance between ADAMTS13 activity and VWF antigen is associated with organ dysfunction and death in patients with systemic inflammation. Despite the natural antithrombotic activity of ADAMTS13, its role in COVID-19 pathophysiology, specifically thrombotic outcomes has not yet been investigated. A thorough understanding of VWF-ADAMTS13 interactions during early and advanced phases of COVID19 could help define the pathophysiology, guide thromboprophylaxis and treatment and improve clinical prognosis.
\end{abstract}




\section{Keywords}

COVID-19

Thrombosis

Inflammation

ADAMTS13

Von Willebrand Factor 


\section{Introduction}

Coronavirus disease of 2019 (COVID-19) is a respiratory illness caused by a novel coronavirus, severe acute respiratory syndrome coronavirus 2 (SARS-CoV-2). SARS-CoV-2 is an enveloped, positive-sense single-stranded RNA virus belonging to Coronaviridae family (1). The COVID-19 outbreak started in Wuhan, China, in late 2019 and rapidly spread to rest of the world. On March 11, 2020, the World Health Organization declared COVID-19 outbreak as pandemic. As of May 13, 2020, the global number of COVID-19 cases stood at 4.32 million with 295,000 deaths (Source: Johns Hopkins Coronavirus Resource Center, https://coronavirus.jhu.edu/). While some SARS-CoV-2 infections are asymptomatic, most patients develop mild symptoms including mild fever, dry cough, sore throat, shortness of breath and muscle pain are present (2-4). In susceptible individuals, the disease progresses to pneumonia, hypoxemia, acute respiratory distress, multi-organ dysfunction that may lead to death (3). The predominance of asymptomatic or mild infections has contributed to the to the rapid spread of COVID-19 compared to earlier coronavirus outbreaks of severe acquired respiratory syndrome (SARS) and Middle East respiratory syndrome (MERS) in 2002 and 2012 respectively $(4,5)$.

\section{Consumptive Coagulopathy and the High Incidence of Thrombosis in COVID-19 Patients}

Altered coagulation is a common feature of acute systemic diseases, specifically to those affecting primarily the respiratory system. Based on studies in patients with acute respiratory distress syndrome, the coexistence of disseminated intravascular coagulation with subsequent consumption of pro-coagulation proteins and platelets has been consistently described (6). This in turn leads to the formation of micro-thrombi in the vascular bed of organs resulting from excess coagulation byproducts and suppression of endogenous anti-coagulation factors (7). The 
coexistence of consumptive coagulopathy and thrombosis are the result of a common pathologic pathway; however the exact mechanisms that tilts the balance towards thrombosis in COVID-19 are less well understood (8). In this sense the clinical presentation described for COVID-19 abnormal coagulation is not unique to this disease, however the magnitude of the thrombotic response and its impact on mortality suggests the presence of additional mechanisms, beyond what is known for similar respiratory acute inflammatory diseases.

Several studies have reported a consistent picture of altered coagulation in severe COVID-19 patients (9). In a study evaluating 449 severe COVID-19 patients, Tang et al. (10) reported positive correlation of 28-day mortality with fibrin degradation product, D-dimers and prothrombin time (PT) and negative correlation with platelet count. Laboratory parameters were recorded at the time of onset of severe COVID-19 (meeting one of the following criteria: respiratory rate $\geq 30$ breaths/min, arterial oxygen saturation $\leq 93 \%$ at rest or $\mathrm{PaO} 2 / \mathrm{FiO} 2 \leq 300$ $\mathrm{mm} \mathrm{Hg}$ ) in the study. In an earlier study comprising of 183 patients, Tang et al. (11) reported elevated D-dimer levels and fibrin degradation product (FDP) levels and prolonged PT and activated partial thromboplastin times (aPTT) at the time of admission in non-survivors compared to survivors. In the same study, significantly lower levels of fibrinogen and antithrombin levels were observed during the late hospitalization in non-survivors. Huang et al. (12) reported higher D-dimers and prolonged PT at the time of admission in ICU-patients compared to non-ICU patients in a study of 41 patients. Wang et al. (13) reported elevated PT in a study of 138 patients. In the same study, elevated levels of D-dimers were found in ICU-patients compared to non-ICU patients as well as in survivors compared to non-survivors in a sub-group of patients with a definitive outcome. In a study of 94 COVID-19 patients, Han et al. (14) reported lower antithrombin and higher D-dimers, FDP and fibrinogen levels compared to 
healthy controls. Zhou et al. (15) reported an association of elevated D-dimers with in-hospital death in a study of 191 patients. Also, elevated PT and decreased platelet counts were observed in non-survivors compared to survivors. Elevated levels of D-dimers were reported by Richardson et al. (16) among 5700 patients in the New York City area. Ranucci et al. (17) reported a procoagulant profile in sixteen patients characterized by increased clot strength by viscoelastography, elevated D-dimer levels and hyperfibrinogenemia. A meta-analysis of 9 studies encompassing 1779 patients with severe disease has identified significantly lower platelet counts (18). A sub-group analysis based on survival has identified even lower platelet counts in non-survivors in this study. Llitzos et al. (19) and Helms et al. (7) reported elevated D-dimer and fibrinogen levels in 26 and 150 ICU-admitted patients respectively. Overall, elevated PT, increased D-dimer and fibrinogen levels, and thrombocytopenia are frequently reported in COVID-19 patients. However, bleeding events requiring therapeutic intervention are not reported.

Multiple studies have reported a higher incidence of thrombotic events, particularly pulmonary embolism, as a frequent complication in COVID-19 patients. Llitzos et al. (19) reported overall rate of $69 \%$ venous thromboembolism (VTE) in ICU-admitted patients. In this study, VTE incidence was found to be significantly higher in patients treated with prophylactic anticoagulation compared to those treated with therapeutic anticoagulation. Helms et al. (7) reported clinically relevant thrombotic complications in 64 out of 150 ICU-admitted patients. Importantly, the incidence of thrombotic complications in COVID-19 acute respiratory distress syndrome (ARDS) patients was significantly higher than non-COVID-19 ARDS patients in this study. Similarly, Klok et al. (20) and Cui et al. (21) also reported higher VTE incidence (31 and $25 \%$ respectively) in ICU-admitted patients. For comparison, in a study by Zhang et al., the 
reported cumulative incidence of VTE in ICU-admitted patients receiving guidelinerecommended thromboprophylaxis was $9.55 \%$ (95\% confidence interval: 6.55-13.81) (22).

A high incidence of disseminated intravascular coagulation (DIC) diagnosed by D-dimer, fibrinogen and antithrombin III levels has become a focus for the initiation of anti-coagulation therapy in severe COVID-19 patients (23), with some studies relying on D-dimers alone (10, 24). A retrospective analysis of 183 patients performed by Tang et al. (11) suggested that more than $70 \%$ of severe COVID-19 patients who succumb to the infection meet the International Society on Thrombosis and Haemostasis (ISTH) definition of DIC. Subsequently Tang et al. (10) reported an equivalent 28 -day mortality rate (30\%) in 99 patients receiving low molecular weight or unfractionated heparin for 7 days compared to 350 non-heparin treated patients or those receiving a less than 7-day course of therapy. A case series reported by Wang et al. (23) detailed the use and outcome following tissue plasminogen activator (tPA) in 3 patients with ARDS and coagulopathy consistent with DIC. Intravenous dosing with tPA indicated a potential benefit in each of the three cases of COVID-19. However, this study also warns of both unrelated effects and high risk of severe bleeding secondary to off-label tPA use. Several of the studies in coagulopathic COVID-19 patients suspected of DIC rely heavily on analysis of fibrin degradation and D-dimer levels, which are expected to be increased during DIC, arterial and venous thromboses, strokes and thrombotic microangiopathies (25). Nonetheless, fibrin accumulation and lysis continuously occur during non-thrombotic inflammation and therefore, significant D-dimer elevations also accumulate during cancers (26) and infections, consistent with inflammatory processes that coincide with the progression of severe COVID-19 related macrophage activation syndrome (27). To date the use of thrombin, plasmin or simultaneous thrombin/plasmin generation assays have not been reported within the context of hemostasis 
management of COVID-19 patients. Since their introduction thrombin and plasmin generation assays have been highly informative regarding the assessment of coagulation and fibrinolysis, impairment of these systems and provide useful guidance need for and monitoring of therapeutic interventions $(28,29)$.

These studies present a heterogeneous picture that is difficult to evaluate in the aggregate. Inclusion criteria for patients varied across these studies, making direct comparisons between the studies difficult. Further, the studies used different regimens of thromboprophylaxis, which could impact outcomes. In some studies, a high proportion of patients were still hospitalized at the end of the reporting period; conclusions and clinical courses therefore were based on incomplete information, and completion of these patients' clinical course could alter the final conclusions. The picture of coagulopathy in COVID-19 is complex. Specific, sensitive and temporal assessments of coagulation and fibrinolysis should be established and further work is needed to untangle the roles of the host inflammatory response, pre-existing thrombotic risk, and prehospitalization pharmacologic regimens in the optimal management of coagulopathy in the setting of COVID-19.

\section{Inflammation, Liver Injury and Hypoxia in COVID-19 Patients}

The risk of hospitalization, morbidity, and mortality from COVID-19 is highest for older patients with preexisting conditions such as hypertension, diabetes, cardiovascular disease and obesity $(12,13,15,16,30,31)$. A common theme of all these co-morbidities is their association with vascular inflammation and endothelial dysfunction $(32,33)$. Pro-inflammatory conditions affect hemostasis by blocking of fibrinolysis and induction of prothrombotic conditions through activation of endothelial cells and innate immune cells via release several factors including tissue factor, von Willebrand Factor (VWF) and neutrophil extracellular traps (NETs) that promote 
thrombosis (34). Induction of pro-inflammatory conditions was reported in the pathophysiology of several viral diseases including flu and SARS (35). Increased inflammation is commonly observed in COVID-19 patients, while severe cases are characterized by immune dysregulation and hyper-inflammation, with a markedly increased serum IL-6 (36). Cytokine release syndrome (CRS) has also been reported in COVID-19 patients and correlates with adverse clinical outcomes (37). The presence of several inflammatory markers such as C-reactive protein (CRP), procalcitonin, ferritin, and fibrinogen are often reported in COVID-19 patients $(12,13,15,16$, 30, 31, 38, 39). In addition, Huang et al. (12) reported elevated levels of IL-2, IL-7, IL-10, granulocyte-colony stimulating factor, interferon- $\gamma$ inducible protein 10 , monocyte chemoattractant protein 1 , macrophage inflammatory protein $1-\alpha$, and tumor necrosis factor- $\alpha$ in ICU-admitted patients. The role of IL-6, in particular, is considered central in the pathogenesis of COVID-19 complications (40), and therefore tocilizumab, an IL-6 inhibitor, is being used in ongoing clinical trials to prevent catastrophic inflammation (41-44).

Liver injury in COVID-19 patients was demonstrated in multiple studies reporting elevated levels of alanine aminotransferase, aspartate aminotransferase and bilirubin $(13,15,16$, 30, 38). The liver is the primary source of plasma proteins, particularly those involved in hemostasis. Thus, liver injury leads to deficiency of several key hemostasis proteins and contributes to coagulopathy (45). Similarly, hypoxemia observed in COVID-19 patients induces prothrombotic conditions through upregulation of plasminogen activator inhibitor and stimulation of endothelial synthesis of pro-coagulants, including tissue factor and VWF (46-49). Thus, multiple clinical characteristics observed in COVID-19 patients contribute to altered coagulation and lead to increased incidence of thrombosis. However, the early onset of 
coagulopathy - before systemic organic effects occur - suggests pro-inflammatory conditions as the primary driving cause of thrombotic events in COVID-19 patients.

\section{Role of VWF-ADAMTS13 metabolism in Hemostasis and Thrombosis}

VWF and its cleaving protease, ADAMTS13, play an important role in the maintenance of hemostasis in microvasculature (50). VWF is a large multimeric glycoprotein primarily expressed by endothelial cells and platelets. Endothelial cells show both basal secretion and regulated release of VWF stored in Weibel-Palade bodies in response to various stimuli. On the other hand, platelets secrete VWF stored in alpha-granules only upon activation (51).

ADAMTS13 is expressed both by hepatic stellate cells and endothelial cells lining vasculature ; the relative relative contribution of hepatic and microvascular expression is not clear (52). ADAMTS13 regulates the biological activity of VWF by cleaving pro-thrombotic ultra-large VWF multimers $(>10,000 \mathrm{kDa})$ secreted from endothelial cells into hemostatically active high molecular weight multimers $(<10,000 \mathrm{kDa})$ under shear stress conditions $(53)$. Severe deficiency of ADAMTS13 results in accumulation of ultra-large VWF multimers leading to microvascular thrombosis and consumptive thrombocytopenia, a condition termed thrombotic thrombocytopenic purpura (TTP) (50). In the event of vascular injury, VWF facilitates binding of platelets to sub-endothelium through its interactions with collagen, thereby inducing thrombus formation (50). Abnormal VWF-ADAMTS13 ratios are implicated in arterial thrombosis (54), ischemic stroke $(55,56)$ pediatric stroke $(57)$ and perioperative thrombosis in infants $(58)$. In this study(58), we identified an imbalance in the proportional ratio of VWF/ADAMTS13, having increased activity and concentration of VWF without a concomitant increase in plasma activity of ADAMTS13; this imbalance is associated with thrombosis. Neonates exposed to severe hypoxia and acidosis demonstrated a higher increase in VWF during cardiac surgery and were at 
higher risk of thrombosis (58). In addition abnormal VWF/ADAMTS13 metabolism has been positively associated with myocardial infarction in young women (59).

Elevated levels of VWF are found in several inflammatory and metabolic disorders including diabetes, obesity and sickle cell disease (60). In patients with systemic inflammatory response syndrome, active VWF predicted 28-day mortality (61). VWF is an acute-phase response protein released by activated endothelial cells in response to inflammatory stimuli (60). Inflammatory cytokines, IL- 8 and TNF- $\alpha$ induced the release of VWF from human umbilical vein endothelial (HUVEC) cells (62). VWF released in inflammation binds to NETs released from activated neutrophils and recruits platelets and leukocytes to promote thrombosis (60).

In patients with systemic inflammation, ADAMTS13 activity decreases proportional to the inflammatory response; an imbalance between ADAMTS13 activity and VWF antigen is associated with organ dysfunction and death $(63,64)$. An interesting observation is that smoking, which is associated with adverse outcomes in COVID-19 patients (65) was also found to be associated with decreased plasma ADAMTS13 levels in a study of 3244 individuals (66). Increased expression of ACE-2, the entry receptor for SARS-CoV-2, in the small airway epithelia of smokers was suggested as the potential mechanism for increased risk of severe COVID-19 in smokers (67). Smoking is also associated with increased inflammatory markers (68).

The imbalance between ADAMTS13 activity and VWF antigen in inflammation is a result of inhibition and/or deficiency of ADAMTS13 activity (69). The inhibition of VWF cleavage by ADAMTS13 in inflammatory conditions was suggested to be mediated by several mechanisms: 1) thrombospondin-1 released from $\alpha$-granules of activated platelets by binding to A2-A3 domain of VWF (70); 2) $\alpha$-defensins released from neutrophils by binding to A2 domain 
of VWF (71); and 3) oxidation of Met 1606 residue in the ADAMTS13 cleavage site of VWF (72). Also, non-physiological high concentrations of IL-6 inhibited cleavage of VWF by ADAMTS13 in in vitro flowing conditions (62). Granulocyte elastases, plasmin, and thrombin that are elevated in inflammatory conditions lower ADAMTS13 activity through its proteolytic cleavage $(73,74)$.

\section{VWF-ADAMTS13 Interactions in COVID-19}

Despite playing an important role in the maintenance of hemostasis and prevention of undesirable thrombosis, VWF-ADAMTS13 interactions haven't received much attention in the evaluation of COVID-19 pathophysiology, specifically VTE. Importantly, reduced ADAMTS13 activity has been shown to correlate with increased inflammation in multiple systems (75-77), while IL-6 has been shown to inhibit the cleavage of ultra-large VWF strings by ADAMTS13 under flowing conditions $(62,78)$. The authors could find only two studies evaluating VWF in COVID-19 patients in literature $(7,79)$. Both studies reported $>3$-fold increase in both VWF antigen and activity in COVID-19 patients. No studies evaluating ADAMTS13 in COVID-19 could be found. Given the prevalence of inflammatory conditions and high VTE incidence in COVID-19, a thorough evaluation of VWF-ADAMTS13 interactions in COVID-19 disease progression is warranted. Overall we propose a mechanistic model in which the systemic release of pro-inflammatory mediators during the severe phase of COVID-19 inhibit the cleavage of high molecular weight VWF or interfere with the proteolytic interaction with ADAMTS13, leading to thrombosis, see Figure 1. The extent of ADAMTS13 suppression needs to be confirmed by studying plasma levels and activity in patients during early and late phases of COVID-19 disease. A thorough understanding of VWF-ADAMTS13 interactions in COVID-19 
could help better define the pathophysiology, clarify the pathogenesis of VTE, improve prediction of clinical prognosis, and better guide thromboprophylaxis and treatment.

\section{Acknowledgements}

This work was partly supported by funds from the Hemostasis Branch/Division of Plasma Protein Therapeutics/Office of Tissues and Advanced Therapies/Center for Biologics Evaluation and Research of the U.S. Food and Drug Administration.

\section{Conflict of interest}

Dr. Buehler reports personal fees from Kalocyte Inc., outside the submitted work. The rest of the authors has nothing to disclose. 


\section{References}

1. Lu R, Zhao X, Li J, et al. Genomic characterisation and epidemiology of 2019 novel coronavirus: implications for virus origins and receptor binding. The Lancet 2020; 395(10224): 565-74.

2. Novel Coronavirus Pneumonia Emergency Response Epidemiology T. [The epidemiological characteristics of an outbreak of 2019 novel coronavirus diseases (COVID-19) in China]. Zhonghua Liu Xing Bing Xue Za Zhi 2020; 41(2): 145-51.

3. Singhal T. A Review of Coronavirus Disease-2019 (COVID-19). Indian J Pediatr 2020; 87(4): 281-6.

4. Rothe $C$, Schunk M, Sothmann P, et al. Transmission of 2019-nCoV Infection from an Asymptomatic Contact in Germany. N Engl J Med 2020; 382(10): 970-1.

5. Petrosillo N, Viceconte G, Ergonul O, et al. COVID-19, SARS and MERS: are they closely related? Clin Microbiol Infect 2020.

6. Gando S, Fujishima S, Saitoh D, et al. The significance of disseminated intravascular coagulation on multiple organ dysfunction during the early stage of acute respiratory distress syndrome. Thrombosis Research 2020; 191: 15-21.

7. Helms J, Tacquard C, Severac F, et al. High risk of thrombosis in patients with severe SARS-CoV-2 infection: a multicenter prospective cohort study. Intensive Care Med 2020.

8. Boral BM, Williams DJ, Boral LI. Disseminated Intravascular Coagulation. American Journal of Clinical Pathology 2017; 146(6): 670-80.

9. Willyard C. Coronavirus blood-clot mystery intensifies. Nature 2020.

10. Tang N, Bai $\mathrm{H}$, Chen $\mathrm{X}$, et al. Anticoagulant treatment is associated with decreased mortality in severe coronavirus disease 2019 patients with coagulopathy. Journal of Thrombosis and Haemostasis 2020; 18(5): 1094-9.

11. Tang N, Li D, Wang $\mathrm{X}$, et al. Abnormal coagulation parameters are associated with poor prognosis in patients with novel coronavirus pneumonia. Journal of Thrombosis and Haemostasis 2020; 18(4): 844-7.

12. Huang C, Wang $\mathrm{Y}$, Li X, et al. Clinical features of patients infected with 2019 novel coronavirus in Wuhan, China. The Lancet 2020; 395(10223): 497-506.

13. Wang D, Hu B, Hu C, et al. Clinical Characteristics of 138 Hospitalized Patients With 2019 Novel Coronavirus-Infected Pneumonia in Wuhan, China. JAMA 2020; 323(11): 1061-9.

14. Han H, Yang L, Liu R, et al. Prominent changes in blood coagulation of patients with SARS-CoV-2 infection. 2020(0): 20200188.

15. Zhou F, Yu T, Du R, et al. Clinical course and risk factors for mortality of adult inpatients with COVID-19 in Wuhan, China: a retrospective cohort study. The Lancet 2020; 395(10229): 1054-62.

16. Richardson S, Hirsch JS, Narasimhan M, et al. Presenting Characteristics, Comorbidities, and Outcomes Among 5700 Patients Hospitalized With COVID-19 in the New York City Area. JAMA 2020.

17. Ranucci M, Ballotta A, Di Dedda U, et al. The procoagulant pattern of patients with COVID-19 acute respiratory distress syndrome. Journal of Thrombosis and Haemostasis; $n / a(n / a)$.

18. Lippi G, Plebani M, Henry BM. Thrombocytopenia is associated with severe coronavirus disease 2019 (COVID-19) infections: A meta-analysis. Clin Chim Acta 2020; 506: 145-8.

19. Llitjos J-F, Leclerc $M$, Chochois $C$, et al. High incidence of venous thromboembolic events in anticoagulated severe COVID-19 patients. Journal of Thrombosis and Haemostasis; n/a(n/a).

20. Klok FA, Kruip M, van der Meer NJM, et al. Incidence of thrombotic complications in critically ill ICU patients with COVID-19. Thromb Res 2020.

21. Cui S, Chen S, Li X, et al. Prevalence of venous thromboembolism in patients with severe novel coronavirus pneumonia. J Thromb Haemost 2020. 
22. Zhang C, Zhang Z, Mi J, et al. The cumulative venous thromboembolism incidence and risk factors in intensive care patients receiving the guideline-recommended thromboprophylaxis. Medicine (Baltimore) 2019; 98(23): e15833.

23. Wang J, Hajizadeh N, Moore EE, et al. Tissue Plasminogen Activator (tPA) Treatment for COVID19 Associated Acute Respiratory Distress Syndrome (ARDS): A Case Series. J Thromb Haemost 2020.

24. Zhang L, Yan X, Fan Q, et al. D-dimer levels on admission to predict in-hospital mortality in patients with Covid-19. J Thromb Haemost 2020.

25. Urban K, Kirley K, Stevermer JJ. PURLs: It's time to use an age-based approach to D-dimer. J Fam Pract 2014; 63(3): 155-8.

26. Schafer M, Werner S. Cancer as an overhealing wound: an old hypothesis revisited. Nat Rev Mol Cell Biol 2008; 9(8): 628-38.

27. Merad M, Martin JC. Pathological inflammation in patients with COVID-19: a key role for monocytes and macrophages. Nat Rev Immunol 2020.

28. Hemker HC, Giesen P, Al Dieri R, et al. Calibrated automated thrombin generation measurement in clotting plasma. Pathophysiol Haemost Thromb 2003; 33(1): 4-15.

29. Simpson ML, Goldenberg NA, Jacobson $L$, et al. Simultaneous thrombin and plasmin generation capacities in normal and abnormal states of coagulation and fibrinolysis in children and adults. Thromb Res 2011; 127(4): 317-23.

30. Goyal P, Choi JJ, Pinheiro LC, et al. Clinical Characteristics of Covid-19 in New York City. New England Journal of Medicine 2020.

31. Ruan Q, Yang K, Wang W, et al. Clinical predictors of mortality due to COVID-19 based on an analysis of data of 150 patients from Wuhan, China. Intensive Care Med 2020.

32. Petrie JR, Guzik TJ, Touyz RM. Diabetes, Hypertension, and Cardiovascular Disease: Clinical Insights and Vascular Mechanisms. Can J Cardiol 2018; 34(5): 575-84.

33. Milan-Mattos JC, Anibal FF, Perseguini NM, et al. Effects of natural aging and gender on proinflammatory markers. Braz J Med Biol Res 2019; 52(9): e8392.

34. Engelmann B, Massberg S. Thrombosis as an intravascular effector of innate immunity. Nature Reviews Immunology 2013; 13(1): 34-45.

35. Jamilloux $Y$, Henry $T$, Belot $A$, et al. Should we stimulate or suppress immune responses in COVID-19? Cytokine and anti-cytokine interventions. Autoimmunity Reviews 2020: 102567.

36. Giamarellos-Bourboulis EJ, Netea MG, Rovina N, et al. Complex Immune Dysregulation in COVID19 Patients with Severe Respiratory Failure. Cell Host Microbe 2020.

37. Zhang C, Wu Z, Li JW, et al. The cytokine release syndrome (CRS) of severe COVID-19 and Interleukin-6 receptor (IL-6R) antagonist Tocilizumab may be the key to reduce the mortality. Int J Antimicrob Agents 2020: 105954.

38. Guan W-j, Ni Z-y, Hu Y, et al. Clinical Characteristics of Coronavirus Disease 2019 in China. New England Journal of Medicine 2020; 382(18): 1708-20.

39. Zhu J, Ji P, Pang J, et al. Clinical characteristics of 3,062 COVID-19 patients: a meta-analysis. J Med Virol 2020.

40. Chen X, Zhao B, Qu Y, et al. Detectable serum SARS-CoV-2 viral load (RNAaemia) is closely correlated with drastically elevated interleukin 6 (IL-6) level in critically ill COVID-19 patients. Clin Infect Dis 2020.

41. Tocilizumab in COVID-19 Pneumonia (TOCIVID-19). Available at: https://ClinicalTrials.gov/show/NCT04317092.

42. Tocilizumab for Prevention of Respiratory Failure in Patients With Severe COVID-19 Infection. Available at: https://ClinicalTrials.gov/show/NCT04377659.

43. Efficacy of Early Administration of Tocilizumab in COVID-19 Patients. Available at: https://ClinicalTrials.gov/show/NCT04346355. 
44. Tocilizumab in the Treatment of Coronavirus Induced Disease (COVID-19). Available at: https://ClinicalTrials.gov/show/NCT04335071.

45. Kujovich JL. Coagulopathy in liver disease: a balancing act. Hematology Am Soc Hematol Educ Program 2015; 2015: 243-9.

46. Mojiri A, Nakhaii-Nejad M, Phan W-L, et al. Hypoxia Results in Upregulation and De Novo Activation of Von Willebrand Factor Expression in Lung Endothelial Cells. Arteriosclerosis, Thrombosis, and Vascular Biology 2013; 33(6): 1329-38.

47. Matsuura Y, Yamashita A, Iwakiri T, et al. Vascular wall hypoxia promotes arterial thrombus formation via augmentation of vascular thrombogenicity. Thromb Haemost 2015; 114(1): 158-72.

48. Ogawa S, Clauss M, Kuwabara K, et al. Hypoxia induces endothelial cell synthesis of membraneassociated proteins. Proceedings of the National Academy of Sciences 1991; 88(21): 9897-901.

49. Fearns C, Loskutoff DJ. Induction of plasminogen activator inhibitor 1 gene expression in murine liver by lipopolysaccharide. Cellular localization and role of endogenous tumor necrosis factor-alpha. Am J Pathol 1997; 150(2): 579-90.

50. Katneni UK, Ibla JC, Hunt R, et al. von Willebrand factor/ADAMTS-13 interactions at birth: implications for thrombosis in the neonatal period. Journal of Thrombosis and Haemostasis $2019 ; 17(3)$ : 429-40.

51. Bryckaert M, Rosa JP, Denis CV, et al. Of von Willebrand factor and platelets. Cell Mol Life Sci 2015; 72(2): 307-26.

52. Zheng XL. ADAMTS13 and von Willebrand factor in thrombotic thrombocytopenic purpura. Annu Rev Med 2015; 66: 211-25.

53. Stockschlaeder M, Schneppenheim R, Budde U. Update on von Willebrand factor multimers: focus on high-molecular-weight multimers and their role in hemostasis. Blood Coagul Fibrinolysis 2014; 25(3): 206-16.

54. Sonneveld MAH, de Maat MPM, Leebeek FWG. Von Willebrand factor and ADAMTS13 in arterial thrombosis: a systematic review and meta-analysis. Blood Reviews 2014; 28(4): 167-78.

55. Bongers TN, de Maat MP, van Goor ML, et al. High von Willebrand factor levels increase the risk of first ischemic stroke: influence of ADAMTS13, inflammation, and genetic variability. Stroke 2006; 37(11): 2672-7.

56. Sonneveld MA, de Maat MP, Portegies ML, et al. Low ADAMTS13 activity is associated with an increased risk of ischemic stroke. Blood 2015; 126(25): 2739-46.

57. Lambers $M$, Goldenberg NA, Kenet $G$, et al. Role of reduced ADAMTS13 in arterial ischemic stroke: a pediatric cohort study. Ann Neurol 2013; 73(1): 58-64.

58. Hunt R, Hoffman CM, Emani S, et al. Elevated preoperative von Willebrand factor is associated with perioperative thrombosis in infants and neonates with congenital heart disease. Journal of Thrombosis and Haemostasis 2017; 15(12): 2306-16.

59. Andersson HM, Siegerink B, Luken BM, et al. High VWF, low ADAMTS13, and oral contraceptives increase the risk of ischemic stroke and myocardial infarction in young women. Blood 2012; 119(6): 1555-60.

60. Gragnano F, Sperlongano S, Golia E, et al. The Role of von Willebrand Factor in Vascular Inflammation: From Pathogenesis to Targeted Therapy. Mediators Inflamm 2017; 2017: 5620314.

61. Hyseni A, Kemperman $\mathrm{H}$, de Lange DW, et al. Active von Willebrand factor predicts 28-day mortality in patients with systemic inflammatory response syndrome. Blood 2014; 123(14): 2153-6. 62. Bernardo A, Ball C, Nolasco L, et al. Effects of inflammatory cytokines on the release and cleavage of the endothelial cell-derived ultralarge von Willebrand factor multimers under flow. Blood 2004; 104(1): 100-6.

63. Bockmeyer $\mathrm{CL}$, Claus RA, Budde U, et al. Inflammation-associated ADAMTS13 deficiency promotes formation of ultra-large von Willebrand factor. Haematologica 2008; 93(1): 137-40. 
64. Reuken PA, Kussmann A, Kiehntopf M, et al. Imbalance of von Willebrand factor and its cleaving protease ADAMTS13 during systemic inflammation superimposed on advanced cirrhosis. Liver Int 2015; 35(1): 37-45.

65. Vardavas $\mathrm{Cl}$, Nikitara K. COVID-19 and smoking: A systematic review of the evidence. Tob Induc Dis 2020; 18: 20.

66. Ma Q, Jacobi PM, Emmer BT, et al. Genetic variants in ADAMTS13 as well as smoking are major determinants of plasma ADAMTS13 levels. Blood Adv 2017; 1(15): 1037-46.

67. Leung JM, Yang CX, Tam A, et al. ACE-2 Expression in the Small Airway Epithelia of Smokers and COPD Patients: Implications for COVID-19. Eur Respir J 2020.

68. Barigye O. Smoking and Inflammation. PLOS Medicine 2005; 2(6): e198.

69. Schwameis M, Schorgenhofer C, Assinger A, et al. VWF excess and ADAMTS13 deficiency: a unifying pathomechanism linking inflammation to thrombosis in DIC, malaria, and TTP. Thromb Haemost 2015; 113(4): 708-18.

70. Wang A, Liu F, Dong N, et al. Thrombospondin-1 and ADAMTS13 competitively bind to VWF A2 and $A 3$ domains in vitro. Thromb Res 2010; 126(4): e260-5.

71. Pillai VG, Bao J, Zander CB, et al. Human neutrophil peptides inhibit cleavage of von Willebrand factor by ADAMTS13: a potential link of inflammation to TTP. Blood 2016; 128(1): 110-9.

72. Chen J, Fu X, Wang Y, et al. Oxidative modification of von Willebrand factor by neutrophil oxidants inhibits its cleavage by ADAMTS13. Blood 2010; 115(3): 706-12.

73. Crawley JTB, Lam JK, Rance JB, et al. Proteolytic inactivation of ADAMTS13 by thrombin and plasmin. Blood 2005; 105(3): 1085-93.

74. Ono T, Mimuro J, Madoiwa S, et al. Severe secondary deficiency of von Willebrand factorcleaving protease (ADAMTS13) in patients with sepsis-induced disseminated intravascular coagulation: its correlation with development of renal failure. Blood 2006; 107(2): 528-34.

75. Liu C, Zhao L, Zhao J, et al. Reduced ADAMTS-13 level negatively correlates with inflammation factors in plasma of acute myeloid leukemia patients. Leuk Res 2017; 53: 57-64.

76. Takaya $\mathrm{H}$, Kawaratani $\mathrm{H}$, Kubo $\mathrm{T}$, et al. Platelet hyperaggregability is associated with decreased ADAMTS13 activity and enhanced endotoxemia in patients with acute cholangitis. Hepatol Res 2018; 48(3): E52-E60.

77. Takaya $\mathrm{H}$, Yoshiji $\mathrm{H}$, Kawaratani $\mathrm{H}$, et al. Decreased activity of plasma ADAMTS13 are related to enhanced cytokinemia and endotoxemia in patients with acute liver failure. Biomed Rep 2017; 7(3): 27785.

78. Chen J, Chung DW. Inflammation, von Willebrand factor, and ADAMTS13. Blood 2018; 132(2): 141-7.

79. Escher R, Breakey N, Lammle B. Severe COVID-19 infection associated with endothelial activation. Thromb Res 2020; 190: 62. 


\section{Figure Legends}

Figure 1: VWF-ADAMTS13 metabolism in inflammation. A) During normal homeostasis, ADAMTS13 regulates the activity of VWF by cleaving pro-thrombotic ultra-large VWF multimers released from endothelial cells in to hemostatically active high molecular weight multimers. B) In inflammatory disorders, pro-inflammatory cytokines (e.g. IL-8 and TNF- $\alpha$ ) stimulate excess release of VWF stored in Weibel-Palade bodies of endothelial cells. VWF interacts with neutrophil extracellular traps (NETs) released from neutrophils to provide a scaffold for platelet adhesion and thrombus formation. C) In inflammation, cleavage of VWF by ADAMTS13 is prevented by multiple mechanisms that either inhibit or reduce the proteolytic activity of ADAMTS13. 


\section{Figure 1}

A] Hemostasis

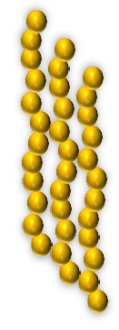

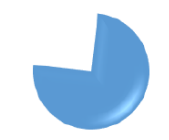

Cleavage by

ADAMTS13

VWF
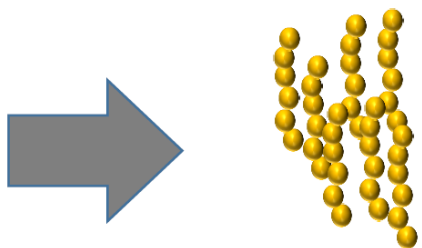

Cleaved VWF

B] Inflammation

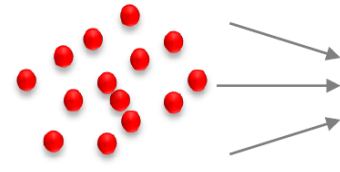

Proinflammatory cytokines

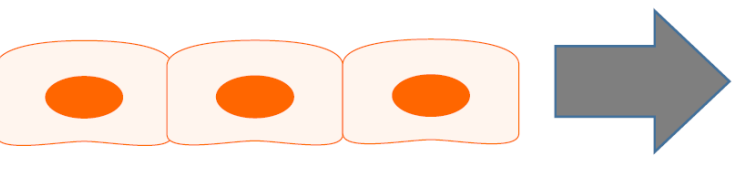

Endothelial cells

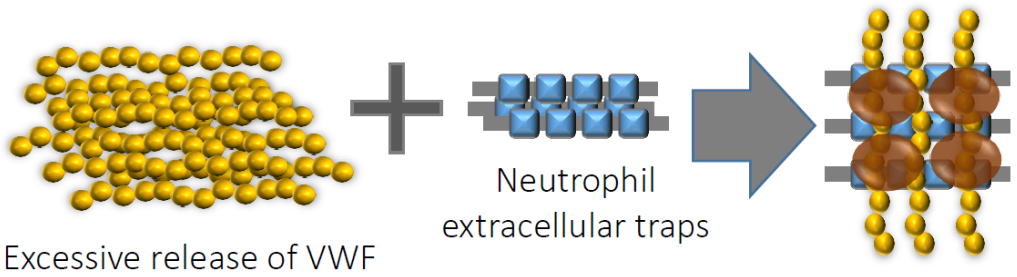

Thrombus

\section{C] Cleavage of VWF by ADAMTS13 is prevented by the following mechanisms}

1) Binding of thrombospondin-1 released from $\alpha$-granules of activated platelets to A2-A3 domain of VWF harboring the proteolytic cleavage site of ADAMTS13

2) Binding of $\alpha$-defensins released from neutrophils to $A 2$ domain of VWF

3) Oxidation of Met 1606 residue in the ADAMTS13 cleavage site of VWF by reactive oxygen species

4) Proteolytic cleavage of ADAMTS13 by granulocyte elastases, plasmin, and thrombin that are elevated in inflammatory conditions 